\title{
Entanglement of bosonic modes of nonplanar molecules
}

\author{
S. Sivakumar \\ Materials Science Division \\ Indira Gandhi Centre for Atomic Research \\ Kalpakkam 603102 INDIA \\ Email: siva@igcar.gov.in \\ Phone: 91-044-27480500-(Extension)22503
}

December 9, 2018

\begin{abstract}
Entanglement of bosonic modes of material oscillators is studied in the context of two bilinearly coupled, nonlinear oscillators. These oscillators are realizable in the vibrational-cumbending motions of $\mathrm{C}-\mathrm{H}$ bonds in dihalomethanes. The bilinear coupling gives rise to invariant subspaces in the Hilbert space of the two oscillators. The dynamics of entanglement and quadrature fluctuations is studied. Two classes of initial conditions, corresponding to the case where the total energy is concentrated in one of the modes and the other wherein the modes share the total energy, in the invariant subspaces are considered. The inadequacy of the known entanglement detection criteria is established and an inseparability criterion that is applicable to the states in the invariant subspaces is provided. Possibility of generating maximally entangled states is indicated.
\end{abstract}

PACS: 03.67.Bg, 03.65.Ca

Keywords: Entanglement, Kerr couplers, local modes 


\section{Introduction}

System with more than one degree of freedom has the potential to get entangled, a feature that is essentially quantal. No classical process has all the features exhibited by the correlations in the entangled quantum states. Such non-classical correlations are essential for quantum teleportation, quantum computation, etc. Hence it is important to identify physical systems wherein quantum entanglement is easily generated, controlled and measured. A host of proposals and demonstrations based on NMR, ion traps, SQUID, photon polarization, etc are known to generate entangled states. These systems can be used as gadgets to perform quantum computation [1, 2]. Recently, the entangling capabilities of molecules and the possibility of engineering their evolution to make quantum gates have been investigated [3, 4]. Molecules have vibrational, rotational and electronic degrees of freedom. Often these degrees of freedom are coupled. It is precisely the coupling among the various degrees of freedom that is exploited to generate suitable molecular states. Study of such systems is important as the vibrational modes perform better as controllable qubits [4]. In addition to the aforementioned degrees of freedom, a nonlinear molecule like the dihalomethane has bending motion. Dihalomethanes are obtained by replacing two of the hydrogen atoms in $\mathrm{CH}_{4}$ with halogen atoms; $\mathrm{CH}_{2} \mathrm{Cl}_{2}$ being an example. These molecules have nonplanar geometrical arrangement of atoms. A classical model to understand the vibrational spectra of these molecules takes the two $\mathrm{C}-\mathrm{H}$ bonds to be two coupled quartic oscillators. The potential energy of the quartic oscillator is

$$
V(x)=\frac{1}{2} M \omega^{2} x^{2}-\alpha x^{4},
$$

where $x$ measures the deviation of $\mathrm{C}-\mathrm{H}$ bond length from its equilibrium value. Here $M$ is the "effective mass" of the $\mathrm{C}-\mathrm{H}$ oscillator. The coefficient $(\alpha)$ of the quartic term is positive. The negative sign for the quartic term is to account for the dissociation of sufficiently excited molecule. Stretching of a C-H bond affects the charge distribution in the molecule. This, in turn, alters the potential of the other $\mathrm{C}-\mathrm{H}$ oscillator, leading to an interaction between the two $\mathrm{C}-\mathrm{H}$ bonds. Thus, the coupling between the bonds is the net effect of mechanical and electromagnetic interactions. A first order perturbation theory is performed to account for the interaction between the bonds. The small parameter $\epsilon$ required for the perturbation theory is $\sqrt{m / M}$, where $m$ is the electron mass. The mass of the hydrogen enters the analysis as the stretching of $\mathrm{C}-\mathrm{H}$ bond stretching involves movement of hydrogen. A detailed discussion of these aspects and a derivation of Hamiltonian operator are given in [5]. The quantum Hamiltonian, obtained after making rotating wave approximation is,

$$
\begin{aligned}
\hat{H} & =\left[\left(\omega-\frac{\gamma}{2}\right)\left(\hat{a}^{\dagger} \hat{a}+\hat{b}^{\dagger} \hat{b}\right)-\frac{\gamma}{2}\left(\hat{a}^{\dagger} \hat{a} \hat{a}^{\dagger} \hat{a}+\hat{b}^{\dagger} \hat{b} \hat{b}^{\dagger} \hat{b}\right)-\epsilon\left(\hat{a}^{\dagger} \hat{b}+\hat{a} \hat{b}^{\dagger}\right)\right], \\
& =\hat{H}_{0}+\hat{H}_{n}+\hat{H}_{c} .
\end{aligned}
$$

This Hamiltonian describes the low energy vibrational states of the molecule correctly. The convention of setting $\hbar=1$ in the expressions is followed in this work. In calculations the actual value of the Plank's constant is used. The Hamiltonian $\hat{H}$ describes a bipartite system of two coupled, 
bosonic oscillators. The operators $\hat{a}^{\dagger}$ and $\hat{a}$ are the creation and annihilation operators for the vibrational mode corresponding to one of the $\mathrm{C}-\mathrm{H}$ oscillators. These operators satisfy the bosonic commutation relation $\left[\hat{a}, \hat{a}^{\dagger}\right]=I$. The corresponding operators for the other C-H oscillator are $\hat{b}^{\dagger}$ and $\hat{b}$. The bending motion is modelled by the term nonlinear in $\hat{a}^{\dagger} \hat{a}$ and $\hat{b}^{\dagger} \hat{b}$. The term with coefficient $\omega-\gamma / 2$ is $H_{0}$, the free Hamiltonian. The nonlinear part is denoted by $\hat{H}_{n}$ and its coefficient is defined by $\gamma=3 \alpha \hbar^{2} / 4 M^{2} \omega^{2}$. The coupling term is $\hat{H}_{i}$ which has $\epsilon=\sqrt{m / M}$ as the coefficient. In the context of nonlinear optics this type of bilinear coupling has models nonlinear couplers for electromagnetic fields [6, 7]. The Hamitlonian has been used in the study of local modes (which are the states of molecules where energy is concentrated in one of the $\mathrm{C}-\mathrm{H}$ bonds in the molecule) and quantum lattice solitons [5]. In the present work, the Hamiltonian $\hat{H}$ is analyzed to study entanglement in the coupled bosonic system. Analysis of bosonic systems in the context of quantum information theory is becoming important [8], especially after the demonstration of teleportation of coherent states of electromagnetic fields $[9$. Theoretical studies have indicated the possibility of generating gates with bosonic modes and the robustness of these gates against thermal dissipation [10].

The Hamiltonian in Eq2 is similar to that used in the analysis of propagation of two-mode electromagnetic fields in a medium with $\chi^{2}$ nonlinearity [11, 12]. The nonlinear medium couples the two modes of the electromagnetic field propagating in it [13. An important difference between the Hamiltonian for a nonlinear coupler and $\hat{H}$ is that the coefficient of the nonlinear term is positive in the former case while it is negative in the later. The Hamiltonian has been investigated in the study of systems for generating maximally entangled states [14, 15], entanglement dynamics [16], control of switching modes [17, generation of bright entangled continuous variable states [18, wavepacket dynamics [19], nonlinear quantum scissors to generate finite dimensional states in systems with infinite dimensional Hilbert space [20] and many more interesting physical applications.

In the case of dihalomethanes, the Hamilotnian describes mechanical oscillators, that is, oscillating masses. There are no external agents, like the Kerr medium in the case of nonlinear couplers, responsible for the coupling between the two oscillators. The coupling is internal and it is not possible to switch off the coupling. The presence of coupling alters the dynamics of the two modes corresponding to the two C-H oscillators significantly. In the present work, the physical properties such as quadrature fluctuations and entanglement are studied. The studies pertain to the situation when dissipation and external fields are absent. Effects of the nonlinearity and the coupling on the dynamics are explored in this work. The results presented here correspond to typical values of $\gamma, \omega$ and $\epsilon$ for a class of dihalomethanes. The relevant values are tabulated in Table. 115. The values of the parameters are nearly equal for the three species. For the purpose of presenting the results, the representative values used for the parameters $\gamma, \epsilon$ and $\omega$ are 125, 30 and 3050 respectively, all expressed in units of $\mathrm{cm}^{-1}$. The organization of the paper is as follows. In Section 2 the special features of the Hamiltonian are discussed. In Section 3, dynamics of entanglement is studied. Known entanglement detection criteria are analyzed in the present context. The inadequacy of these criteria is established and a new criterion suitable for states of the present system is given. 
Table 1: Values of $\gamma, \epsilon$ and $\omega$ expressed in $\mathrm{cm}^{-1}$ units

\begin{tabular}{|l||c|c|l|}
\hline Molecule & $\gamma$ & $\epsilon$ & $\omega$ \\
\hline $\mathrm{CCl}_{2} \mathrm{H}_{2}$ & 127.44 & 29.54 & 3020.1 \\
\hline $\mathrm{CBr}_{2} \mathrm{H}_{2}$ & 125.45 & 32.80 & 3026.8 \\
\hline $\mathrm{CI}_{2} \mathrm{H}_{2}$ & 124.25 & 33.69 & 3068.7 \\
\hline
\end{tabular}

Results on the fluctuations of single-mode and two-mode quadratures are presented in Section 4. In Section 5, it is shown that maximally entangled states are generated at specific instants during evolution from a separable state. Results are summarized in Section 6 ,

\section{Special features of $\hat{H}$}

The presence of the bilinear coupling term $\hat{H}_{c}$ endows the Hamiltonian $\hat{H}$ with interesting features. A suitable basis to expand an arbitrary state of the oscillators is the set $\{|n, m\rangle\}, n, m=0,1,2, \cdots$. The quantum numbers $n$ and $m$ label the states of the oscillators corresponding to the two modes. In the absence of the coupling, for $n+m=N$, then there are $N+1$ eigenstates of the form $|N-m, m\rangle$, with $m$ ranging from 0 to $N$. The corresponding eigenvalues are $\left(\omega-\frac{\gamma}{2}\right) N-\frac{\gamma}{2}\left[(N-m)^{2}+m^{2}\right]$. For odd $N$, there are $(N+1) / 2$ distinct eigenvalues and the states are doubly degenerate. When $N$ is even, there are $(N+2) / 2$ distinct eigenvalues; there is one nondegenerate state $|N / 2, N / 2\rangle$ and the rest are doubly degenerate. When $\epsilon \neq 0$, the degeneracy is lost and the states $|n, m\rangle$, except when $n=m=0$, cease to be eigenstates of $\hat{H}$. The free Hamiltonian $\hat{H}_{0}$, which is the total number operator, commutes with the total Hamiltonian $\hat{H}$ and the coupling term $\hat{H}_{i}$. Consequently, the Hilbert space of the coupled system splits into disjoint, irreducible, invariant subspaces. Each invariant subspace is characterized by total the quantum number $N=n+m$ and the symbol $S_{N}$ is used to represent the corresponding invariant subspace. The subspace $S_{N}$ is the span of the vectors $\{|N-m, m\rangle\}_{m=0}^{N}$ and its dimension is $N+1$. For instance, when $n+m=1$, there are two possible states, namely, $|0,1\rangle$ and $|1,0\rangle$. The span of these two canonical basis states forms the relevant invariant subspace $S_{1}$. If coupling is absent the irreducible, invariant subspaces are of dimension one. The nonlinear and the coupling terms in the Hamiltonian do not commute. Consequently, the factorization of the time-evolution operator is difficult. Nevertheless, including a nonlinear coupling term, namely, $\hat{a}^{\dagger} \hat{a} \hat{b}^{\dagger} \hat{b}$, allows to write the Hamiltonian as a sum of three mutually commuting terms [16. In the present work the discussions are limited to the bilinear coupling model.

Another remarkable feature of the invariant spaces is that the states of the form $|n, m\rangle$ are the only product states in the respective invariant subspaces. Every other state in a given invariant subspaces is entangled. The most general state in the invariant subspace $S_{N}$ is of the form 
$\sum_{n=0}^{N} c_{n}|n, N-n\rangle$. If this is to be a product state, then it should be expressible as $\sum_{n=0}^{N} c_{n} \mid n, N-$ $n\rangle=\sum_{k=0}^{N} g_{k}|k\rangle_{a} \times \sum_{m=0}^{N} f_{m}|m\rangle_{b}$, the suffixes in the states indicating the corresponding modes. Consequently, the coefficients satisfy $c_{n}=g_{n} f_{N-n}$. Let the state of the bipartite system is such that at least two coefficients, say, $c_{p}$ and $c_{q}$ are nonzero. The relation among the coefficients implies that $g_{p}, g_{q}, f_{N-p}$ and $f_{N-q}$ are nonzero. As a result, the product state is $g_{p} f_{N-p}|p, N-p\rangle+$ $g_{q} f_{N-q}|q, N-q\rangle+g_{p} f_{N-q}|p, N-q\rangle+g_{q} f_{N-p}|q, N-p\rangle$. However, the states $|p, N-q\rangle$ and $|q, N-p\rangle$ do not belong to $S_{N}$, their total quantum numbers are $N-q+p$ and $N-p+q$ respectively and not $N$. Hence, it is not possible to express the state with two nonzero $c_{n}$ as a product of states in $S_{N}$. This argument can be extended to states wherein more number of $c_{n}$ are nonzero to show that such states are entangled. If there is only one non-vanishing $c_{n}$, then the $g_{n}$ and $f_{N-n}$ are the only nonzero coefficients. The corresponding state $|n, N-n\rangle$ is a product state.

Explicit construction of the eigenfunctions in terms of the number states and eigenvalues of the Hamiltonian is possible by the method of number states [5]. However, the resulting expressions for the eigenstates are complicated. On treating the coupling as a perturbation, simple expressions for approximate eigenstates and eigenvalues are obtained by first order perturbation theory. Since $\left\langle N-m, m\left|\hat{a}^{\dagger} \hat{b}+\hat{a} \hat{b}^{\dagger}\right| N-m, m\right\rangle$ is zero, the eigenvalues do not change to first order in perturbation. Apart from an overall nonramlization factor, the perturbed eigenstates, denoted with a suffix $p$, are

$$
|N-m, m\rangle_{p} \propto|N-m, m\rangle+f_{1}|N-m+1, m-1\rangle+f_{2}|N-m-1, m+1\rangle
$$

where

$$
\begin{aligned}
& f_{1}=\frac{\epsilon}{\gamma} \frac{\sqrt{(N-m+1) m}}{1+N-2 m} \\
& f_{2}=\frac{\epsilon}{\gamma} \frac{\sqrt{(N-m)(m+1)}}{1-N+2 m}
\end{aligned}
$$

If $|N-2 m|=1$, either $f_{1}$ or $f_{2}$ becomes infinity and the perturbation theory is not applicable. Though the eigenstates are doubly degenerate, the results of nondegenerate perturbation theory are applicable if $|N-2 m| \neq 1$. This holds for all for states except those states of the form $|m, m+1\rangle$ and $|m+1, m\rangle$. The transition matrix element $\left\langle m+1, m\left|\hat{a} \hat{b}^{\dagger}+\hat{a}^{\dagger} \hat{b}\right| m, m+1\right\rangle$ is nonzero and hence the nondegenerate perturbation expansion is invalid. The matrix element of $\hat{a} \hat{b}^{\dagger}+\hat{a}^{\dagger} \hat{b}$ among the other states vanish and the Eqn,4 holds for such states. Further it is required that $\gamma>>\epsilon$ for the perturbation expansion to be valid and this condition holds in the case of dihalomethanes. Two important special cases, $|N, 0\rangle$ and its degenerate counterpart $|0, N\rangle$, are analyzed. Physically, these states correspond to the situation in which the total energy is concentrated in one of the modes and are referred as "local modes". From the expression for perturbed states in Eqn. 4,

$$
\begin{aligned}
|N, 0\rangle_{p} & \propto|N, 0\rangle+\frac{\epsilon}{\gamma} \frac{\sqrt{N}}{N+1}|N-1,1\rangle, \\
|0, N\rangle_{p} & \propto|0, N\rangle+\frac{\epsilon}{\gamma} \frac{\sqrt{N}}{N-1}|1, N-1\rangle,
\end{aligned}
$$


where $N>1$ to ensure that the perturbation results are valid. For large $N$, the second term of the perturbed state is negligible. Consequently, the unperturbed states $|N, 0\rangle$ and $|0, N\rangle$ qualify as approximate eigenstates of the perturbed Hamiltonian $\hat{H}$. The validity of the result is ascertained by studying the overlap between the initial state and the corresponding time-evolved state. Fidelity is defined as the absolute value of overlap of the initial state with the time-evolved state. This definition means that the fidelity is given by the absolute value of the expectation value of the evolution operator $\exp (-i t \hat{H})$ evaluated in the initial state. If a state is an eigenstate of $\hat{H}$ then its fidelity is unity at all times. The fidelity for the states $|2,0\rangle$ and $|1,1\rangle$ are given in given in Fig. 1a. The results are based on nonperturbative evolution of the initial state so that the applicability of the perturbation theory is justified. The explicit construction of the Hamiltonian matrix required for the numerical computation is specified in the next section. The fidelity of the state $|2,0\rangle$ becomes very small, an indication that the state is not an eigenstate. In the same figure the fidelity of the state $|1,1\rangle$ is given. Though the fidelity does not become zero, its deviation from unity is significant as the state is not an eigenstate. In Fig. 1b fidelity of the states $|0,4\rangle$ and $|2,2\rangle$ are given. It is seen that the fidelity of the former remains close to unity for all times. Thus the state $|4,0\rangle$ is an approximate eigenstate. This is expected based on the perturbation theory result that for large $N$ the local modes are approximate eigenstates. The fidelity of the state $|2,2\rangle$ is appreciably different from unity since the state is not a good approximation to an eigenstate. Though the state $|1,3\rangle$ has significant amount of energy concentrated in one of the modes, the total quantum number (equal to 4 in this case) is not sufficient to make it an approximate eigenstate.

The role of nonlinear term in the Hamiltonian deserves to be stressed. For the local modes to be approximate eigenstates the nonlinearity parameter $\gamma$ should be large in comparison to the coupling strength $\epsilon$. In addition, the eigenstates of $\hat{H}$ are all entangled, except the ground state, if nonlinearity is absent or weak. Nonlinearity makes it possible for the system to have approximate eigenstates which are separable, despite the presence of coupling. If $\gamma$ vanishes, first order perturbation theory fails and the states of the form $|n, m\rangle$ do not approximate the eigenstates.

\section{Dynamics of Entanglement}

In the absence of coupling, the Hamiltonian is a function of the two number operators, namely, $\hat{a}^{\dagger} \hat{a}$ and $\hat{b}^{\dagger} \hat{b}$. The states $|N-m, m\rangle$ are eigenstates of the coupling-free Hamiltonian. From the expressions for the perturbed eigenstates given in Eq. 4, it is clear that if the nonlinearity dominates over the coupling, $\gamma>>\epsilon$, the separable states $|N-m, m\rangle$ become the approximate eigenstates of the total Hamiltonian. The other extreme case corresponds to the absence of the nonlinear term while the coupling is retained. Perturbation theory results are not valid in this case. However, in this limit the Hamiltonian $\hat{H}$ is the sum two operators, namely, $\hat{a}^{\dagger} \hat{a}+\hat{b}^{\dagger} \hat{b}$ and $\hat{a}^{\dagger} \hat{b}+\hat{a} \hat{b}^{\dagger}$, which commute. By the Baker-Campbell-Hausdorff formula[24], the unitary time evolution operator $\exp (-i t \hat{H})$ factorises to

$$
\hat{U}=\exp (-i t \hat{H})=\exp \left(i \epsilon t\left(\hat{a}^{\dagger} \hat{b}+\hat{a} \hat{b}^{\dagger}\right)\right) \exp \left(-i \omega t\left(\hat{a}^{\dagger} \hat{a}+\hat{b}^{\dagger} \hat{b}\right)\right) .
$$




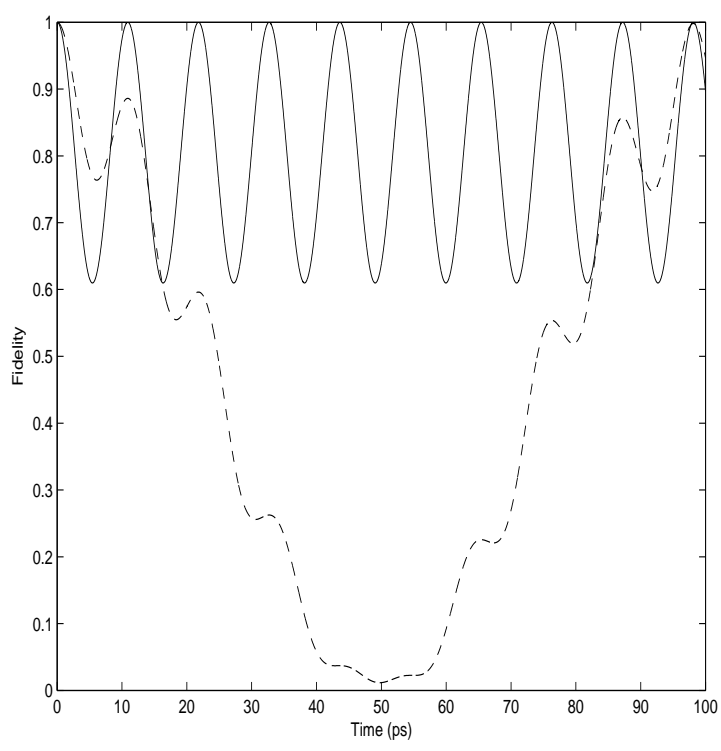

(a) $\mathrm{N}=2$

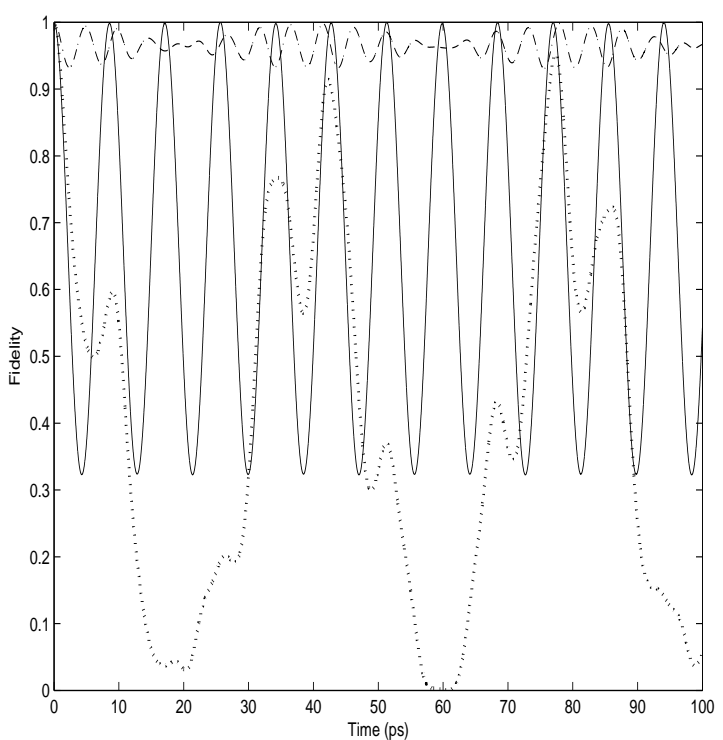

(b) $\mathrm{N}=4$

Figure 1: Fidelity $\langle\exp (-i t \hat{H})\rangle$ of states corresponding to total quantum numbers $N=2$ and $N=4$. The expectation value is evaluated in the states for which fidelity is calculated. Fidelity of $|0,2\rangle$ (dashed) and $|1,1\rangle$ (continuous) are shown in (a). Fidelity of $|0,4\rangle$ (dashed), $|2,2\rangle$ (continuous) and $|1,3\rangle$ (dotted) are shown in (b). Values used for the parameters are $\gamma=125 \mathrm{~cm}^{-1}, \epsilon=30 \mathrm{~cm}^{-1}$ and $\omega=3050 \mathrm{~cm}^{-1}$. 
The time-evolved states are obtained by the action of $\hat{U}$ on the initial states.

For dihalomethanes, the parameters $\gamma$ and $\epsilon$ satisfy $\gamma \approx 4 \epsilon$ (see Table 1). Hence, the advantages of the limiting cases, namely, $\gamma=0$ or $\epsilon=0$, in solving for the dynamics are not available. However, the availability of invariant subspaces simplifies the study of dynamics if the initial state is in one of the invariant subspaces. If the initial state is $\sum_{r=0}^{N} c_{r}|N-r, r\rangle$, the evolved state has no overlap with states in other invariant spaces. Hence, the dynamics of such states is dictated by a truncated Hamiltonian of dimension $N+1$, where $N$ is the total quantum number characterizing the invariant space containing the initial state. The Hamiltonian, expressed in the canonical basis $|n, m\rangle$, is the sum of a diagonal matrix and a non-diagonal matrix. The diagonal matrix is the sum of the free Hamiltonian $\hat{H}_{0}$ and the nonlinear term $\hat{H}_{n}$. The corresponding matrix elements are

$$
\left(\hat{H}_{0}+\hat{H}_{n}\right)_{j, k}=\left[\omega N-\frac{\gamma}{2}\left(N^{2}+N-2 N j+2 j^{2}\right)\right] \delta_{j, k} \quad j, k=0,1,2, \cdots N .
$$

The interaction $\hat{H}_{c}$ is nondiagonal whose elements are

$$
\left(\hat{H}_{c}\right)_{j, j+1}=\sqrt{(j+1)(N-j)} \quad j=1,2, \cdots N-1,
$$

and other elements vanish. This truncated Hamiltonian matrix is sufficient to study the evolution of states belonging to the particular invariant subspace $S_{N}$. This finite size matrix makes it easier to numerically track the evolution of the system. The results given subsequently are based on numerical computations. For the calculations presented here the largest matrix is of order five, used to study states in the invariant subspace $S_{4}$. For an initial condition involving states from two or more invariant subspaces, the Hamiltonian matrix has to be enlarged. It is to be noted that the evolution dictated by $\hat{H}$ is solvable analytically[25]. If $\hat{a}(t)$ and $\hat{b}(t)$ represent time-evolved operators, they are related to the initial operators $\hat{a}$ and $\hat{b}$ through

$$
\left(\begin{array}{c}
\hat{a}(t) \\
\hat{b}(t)
\end{array}\right)=\exp \left(-i \frac{3 \gamma t}{2} \hat{N}\right)\left[\begin{array}{cc}
\exp \left[-i\left(\epsilon-\frac{\gamma}{2} \hat{H}_{i}\right) t\right]+H . c . & \exp \left[-i\left(\epsilon-\frac{\gamma}{2} \hat{H}_{i}\right) t\right]-H . c . \\
\exp \left[-i\left(\epsilon-\frac{\gamma t}{2} \hat{H}_{i}\right) t\right]-H . c . & \exp \left[-i\left(\epsilon-\frac{\gamma t}{2} \hat{H}_{i}\right) t\right]+H . c .
\end{array}\right]\left(\begin{array}{c}
\hat{a} \\
\hat{b}
\end{array}\right)
$$

Here $\hat{H}_{i}=\hat{a}^{\dagger} \hat{b}+\hat{a} \hat{b}^{\dagger}$. The term $\exp \left(i \gamma t \hat{H}_{i} / 2\right)$ does not factorize further. As a consequence, the expressions for expectation values become lengthy and cumbersome. This justifies the use of numerical calculations which are very accurate.

As noted earlier, states of the form $|N-m, m\rangle$ are the only product states in the invariant subspace $S_{N}$. Due to the coupling, the two modes may get entangled during evolution even though the initial state is a product state. Quantification of entanglement between the oscillators is easily done using density operators. If the initial state of the system is in one of the invariant subspaces, say, $S_{N}$, then the density operator for the bipartite system is

$$
\hat{\rho}=\sum_{m=0}^{N} \sum_{n=0}^{N} c_{n} c_{m}^{*}|N-n, n\rangle\langle N-m, m|
$$


The coefficients are time-dependent and satisfy suitable initial conditions. The reduced density operators for the two oscillators are

$$
\hat{\rho}_{a}=\sum_{n=0}^{N}\left|c_{n}\right|^{2}|N-n\rangle\langle N-n|,
$$

and

$$
\hat{\rho}_{b}=\sum_{n=0}^{N}\left|c_{n}\right|^{2}|n\rangle\langle n|
$$

The suffixes $a$ and $b$ indicate the two modes respectively. The reduced density operators are diagonal in their respective number state basis. Further, the diagonal elements of the reduced density operator are the same except for a reversal of ordering: the coefficient $\left|c_{n}\right|^{2}$ appears as the probability for the state $|n\rangle$ for the $a$-mode while it appears as that of the state $|N-n\rangle$ for the other mode. This restriction is only because the state of the bipartite system belongs to the subspace $S_{N}$.

A measure of entanglement in bipartite, pure states is the linear entropy $L$ defined as follows [21]:

$$
L=1-\operatorname{Tr}\left[\hat{\rho}_{a} \hat{\rho}_{a}\right]
$$

The numerical value is independent of whether $\hat{\rho}_{a}$ or $\hat{\rho}_{b}$ is used in the expression. Using the reduced density matrix expression given in Eq. 14, the linear entropy is $1-\sum_{n=0}^{N}\left|c_{n}\right|^{4}$. Another measure of entanglement[21] is von Neuman entropy $S$ defined as

$$
S=\operatorname{Tr}\left[\hat{\rho}_{a} \log \hat{\rho}_{a}\right]
$$

Its value is zero for separable states and reaches the maximum value of $\log _{2} M$ for a system whose Hilbert space is of dimension $M$. The explicit expression for $S$ is $2 \sum_{n=0}^{N}\left|c_{n}\right|^{2} \log _{2}\left|c_{n}\right|$. Nonzero values of entropy ( $S$ or $L$ ) imply that the system is entangled. In Fig. 2 the dynamics of $S$ is shown as the system evolves from initial conditions of the form $|n, m\rangle$ which are separable states and hence the entropy is zero. Instead of plotting $S$, the ratio of $S$ and $\log _{2}(N+1)$ is plotted. The ratio varies from zero to a maximum of unity for any state. This makes it easier to compare the evolution of $S$ for initial states from different invariant subspaces. The evolution of entropy when the total quantum number is one, two and three are shown in Figs. 2a -2c respectively. The entropy increases substantially approaching the maximum attainable entanglement and subsequently oscillates. However, the state $|0,4\rangle$, whose entropy evolution is shown in Fig. 2d, does not evolve to states of high entropy. The entropy of the local mode $|0,4\rangle$ is zero as it is a product state. Being an approximate eigenstate, the evolved states do not differ significantly from the initial state. As a consequence, the entropy remains low during evolution. In practical terms, this implies that local modes are not good candidates for generating states of high entanglement using bilinear coupling. The situation changes drastically if other initial conditions are chosen. When the system evolves 
from initial conditions other than the local modes, the entropy $S$ attains values closer to the maximum allowed value of $\log _{2} N$. Evolution of entanglement when the initial states are not local modes is shown in Fig. 3. In particular, comparison of Fig. 2d for the state $|0,4\rangle$ and Fig. 3(d) for the state $|1,3\rangle$ of same total quantum number, shows the clear distinction between the evolution of a local and a nonlocal mode for $N=4$. The evolution takes the system to maximally entangled states whenever the reduced density operator of the form $\frac{1}{\sqrt{N+1}} \sum_{n=0}^{N}|n\rangle\langle n|$ during evolution.

It is important to note that if the nonlinear term is absent, the bilinear coupling cannot lead to entangled states from separable, initial states unless the initial states are nonclassical[22, 23]. Here "nonclassical" implies that the Glauber-Sudarshan $P$-function for the state in the diagonal coherent state representation does not qualify as a probability density[24, 26]. The states in $S_{N}$ for $N>0$ are non-classical and hence the evolved states are entangled. The presence of the nonlinearity along with the bilinear coupling can entangle states which are classical. In essence, bilinear cross coupling, without the presence of nonlinear term in the Hamiltonian, cannot generate entanglement if the initial states are classical.
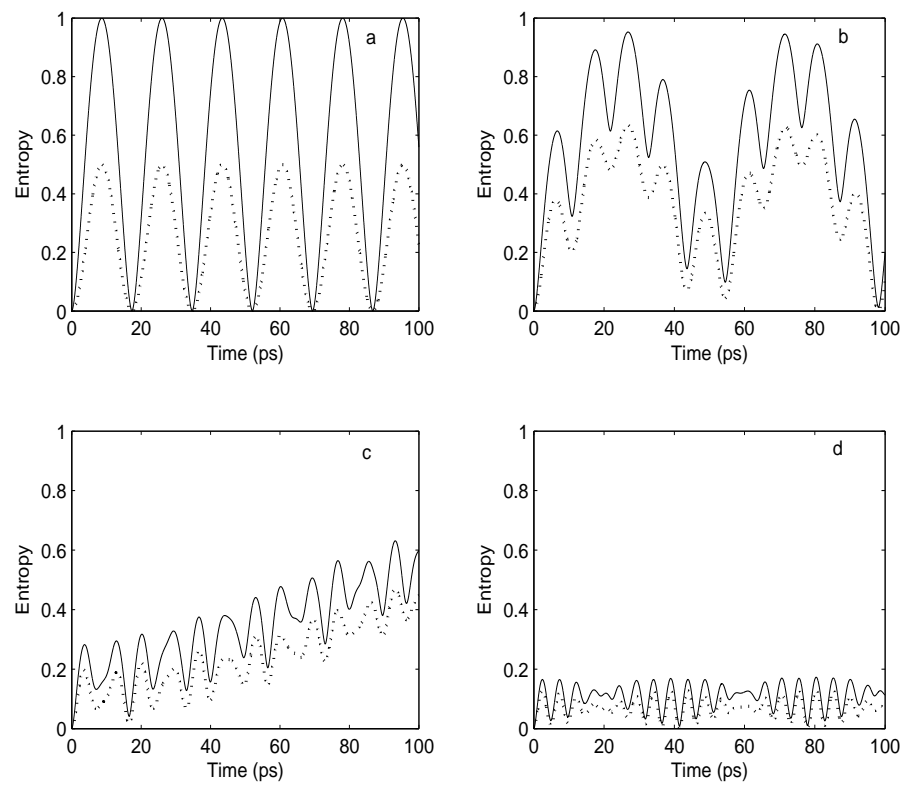

Figure 2: Temporal evolution of von Neuman entropy $S$ and linear entropy $L$. The quantities plotted are $\frac{\operatorname{Tr}\left[\rho_{1} \log \rho_{1}\right]}{\log _{2}(N+1)}$ (continuous curve) and $L$ (dashed curve) for various initial conditions. Figures (a)(d) show the evolution as the bipartite system evolves from the initial conditions $|0,1\rangle,|0,2\rangle,|0,3\rangle$ and $|0,4\rangle$ respectively. Values of $\gamma, \epsilon$ and $\omega$ are as indicated in Fig. 1.

The states under consideration are non-Gaussian, pure states. While von Neumann entropy 

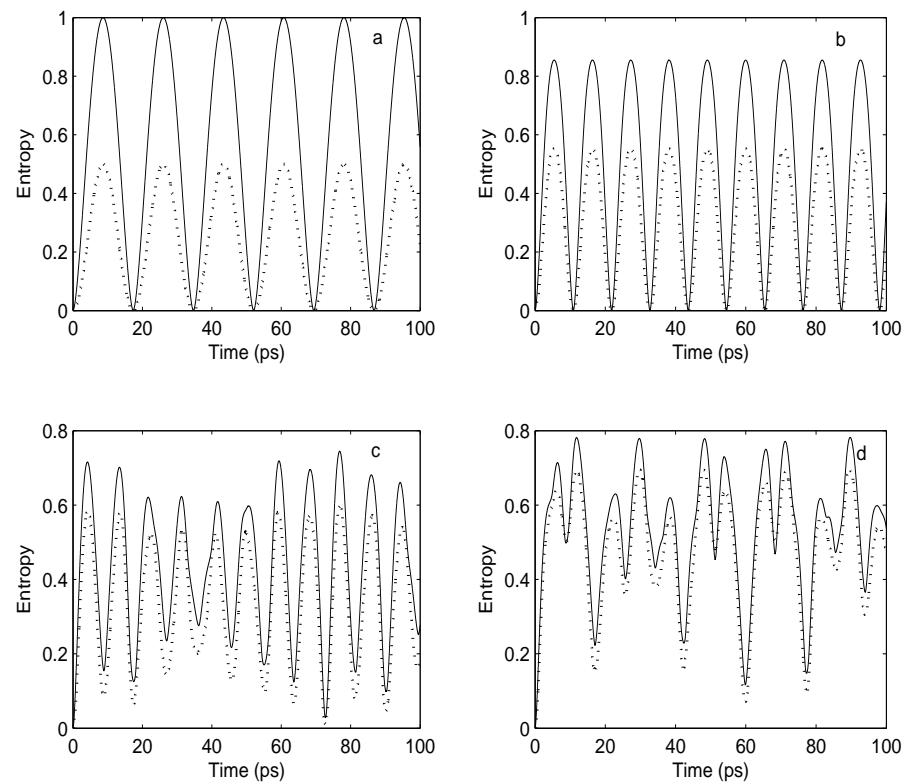

Figure 3: Temporal evolution of von Neumann entropy $S$ and linear entropy $L$. The quantities plotted are $\frac{\operatorname{Tr}\left[\rho_{1} \log \rho_{1}\right]}{\log _{2}(N+1)}$ (continuous curve) and $L$ (dashed curve) for various initial conditions. Figures (a)-(d) show the evolution as the bipartite system evolves from the initial conditions $|1,0\rangle,|1,1\rangle,|1,2\rangle$ and $|1,3\rangle$ respectively. For the state $|1,3\rangle$, the ratio attains values close to 0.8 , indicating the entanglement is nearly 3 ebits. Higher energy states can generate more entanglement. Values of $\gamma, \epsilon$ and $\omega$ are as indicated in Fig. 1. 
provides the unique measure of entanglement for pure states, it is required to have a criterion expressed in terms of measurable quantities like the moments of the creation-, annihilation- and number- operators. Many criteria have been proposed to detect entanglement, but none of them are universally applicable. All these criteria are sufficient conditions for entanglement. For instance, the sufficient and necessary conditions for entanglement in bipartite Gaussian states are known[27, 28]. However, for non-Gaussian states these criteria may not work. In what follows, several of the known criteria are applied to the states in the invariant subspaces. The explicit calculations are simplified by using the result that the expectation value of $\hat{a}^{m} \hat{b}^{n} \hat{a}^{\dagger p} \hat{b}^{\dagger q}$ in the states in any invariant subspace is zero if $m+n \neq p+q$, where $m, n, p$ and $q$ are non-negative integers.

1. Duan et al criterion [27]: This criterion is expressed in terms of uncertainties in the two-mode operators:

$$
\begin{aligned}
& \hat{u}=\frac{1}{\sqrt{2}}\left[|m|\left(\hat{a}+\hat{a}^{\dagger}\right)+\frac{1}{m}\left(\hat{b}+\hat{b}^{\dagger}\right)\right], \\
& \hat{v}=\frac{1}{i \sqrt{2}}\left[|m|\left(\hat{a}-\hat{a}^{\dagger}\right)-\frac{1}{m}\left(\hat{b}-\hat{b}^{\dagger}\right)\right] .
\end{aligned}
$$

Separable states satisfy $\left\langle(\Delta u)^{2}\right\rangle+\left\langle(\Delta v)^{2}\right\rangle>\left(1+m^{4}\right) / m^{2}$. For the states in $S_{N}$, the relation becomes $\left\langle(\Delta u)^{2}\right\rangle+\left\langle(\Delta v)^{2}\right\rangle=\left(|m|^{4}+1\right) / m^{2}+|m|^{2} N_{a}+N_{b} / m^{2}$, where $N_{a}$ and $N_{b}$ refer to the expectation values $\left\langle\hat{a}^{\dagger} \hat{a}\right\rangle$ and $\left\langle\hat{b}^{\dagger} \hat{b}\right\rangle$ respectively. Since the number operators are non-negative operators, the sum of the uncertainties indeed satisfies the condition of separability although the states are entangled as evident from the earlier discussion on the von Neumann entropy. Hence this criterion is not useful in the present context.

2. Mancini et al criterion : This criterion employs the product of uncertainties in the operators considered in the criterion due to Duan et al, choosing $m=1$ [29]. The inequality to be satisfied to identify entangled states is that

$$
\left\langle(\Delta u)^{2}\right\rangle\left\langle(\Delta v)^{2}\right\rangle<1 .
$$

When applied to the states in $S_{N},\left\langle(\Delta u)^{2}\right\rangle=1+\left\langle\hat{a}^{\dagger} \hat{a}+\hat{b}^{\dagger} \hat{b}\right\rangle+\left\langle\hat{a}^{\dagger} \hat{b}+\hat{a} \hat{b}^{\dagger}\right\rangle$ which can be written as $1+\left\langle\left(\hat{a}^{\dagger}+\hat{b}^{\dagger}\right)(\hat{a}+\hat{b})\right\rangle$. The operator $\left(\hat{a}^{\dagger}+\hat{b}^{\dagger}\right)(\hat{a}+\hat{b})$ is of the form $\hat{A}^{\dagger} \hat{A}$, which is positive. Hence, $\left\langle(\Delta u)^{2}\right\rangle>1$. Similarly, $\left\langle(\Delta v)^{2}\right\rangle=1+\left\langle\left(\hat{a}^{\dagger}-\hat{b}^{\dagger}\right)(\hat{a}-\hat{b})\right\rangle>1$. Thus, the product of the uncertainties is larger than unity making the criterion not useful in identifying the entangled states in $S_{N}$.

3. Shchukin-Vogel's criteria:

Many of the known criteria are expressible in terms of the determinants introduced in [30]:

All separable states satisfy

$$
D_{3}=\left|\begin{array}{ccc}
1 & \left\langle\hat{a}^{\dagger}\right\rangle & \left\langle\hat{b}^{\dagger}\right\rangle \\
\langle\hat{a}\rangle & \left\langle\hat{a}^{\dagger} \hat{a}\right\rangle & \left\langle\hat{a}^{\dagger} \hat{b}^{\dagger}\right\rangle \\
\langle\hat{b}\rangle & \langle\hat{a} \hat{b}\rangle & \left\langle\hat{b}^{\dagger} \hat{b}\right\rangle
\end{array}\right| \geq 0 .
$$


This inequality is a stronger version of Duan et al criterion. If the determinant is negative, the state is entangled. For the states in an invariant subspace $S_{N}$, the determinant is explicitly evaluated to give $\left\langle\hat{a}^{\dagger} \hat{a}\right\rangle\left\langle\hat{b}^{\dagger} \hat{b}\right\rangle$ which is positive. Thus, the criterion does not detect entanglement in these states. Another criterion that works for entangled coherent states $|\alpha\rangle|\beta\rangle-|-\alpha\rangle|-\beta\rangle$ is that

$$
\left|\begin{array}{ccc}
1 & \langle\hat{b}\rangle & \left\langle\hat{a} \hat{b}^{\dagger}\right\rangle \\
\left\langle\hat{b}^{\dagger}\right\rangle & \left\langle\hat{b}^{\dagger} \hat{b}\right\rangle & \left\langle\hat{a}^{\dagger} \hat{b}^{\dagger} \hat{b}\right\rangle \\
\left\langle\hat{a}^{\dagger} \hat{b}\right\rangle & \left\langle\hat{a}^{\dagger} \hat{b}^{\dagger} \hat{b}\right\rangle & \left\langle\hat{a}^{\dagger} \hat{a} \hat{b}^{\dagger} \hat{b}\right\rangle
\end{array}\right|<0,
$$

implies entanglement. For the states under consideration in the present work, the determinant is $\left\langle\hat{a}^{\dagger} \hat{a} \hat{b}^{\dagger} \hat{b}\right\rangle\left\langle\hat{b}^{\dagger} \hat{b}\right\rangle$ which is positive and hence inconclusive about the entanglement in the states.

4. $\mathrm{SU}(2)$ criterion: The operators corresponding to the two modes can be combined to obey $\mathrm{SU}(2)$ and $\mathrm{SU}(1,1)$ algebra. The corresponding uncertainty relations provide criteria to detect entanglement. The expression for $\mathrm{SU}(2)$ uncertainty product satisfied by separable states [31, 32] when applied to the states in the invariant subspaces is

$$
\left\langle\hat{a}^{\dagger} \hat{a} \hat{b}^{\dagger} \hat{b}\right\rangle\left[4\left\langle\hat{a}^{\dagger} \hat{a} \hat{b}^{\dagger} \hat{b}\right\rangle+2\left\langle\hat{a}^{\dagger} \hat{a}\right\rangle+2\left\langle\hat{b}^{\dagger} \hat{b}\right\rangle\right]+4\left\langle\hat{a}^{\dagger} \hat{a}\right\rangle\left\langle\hat{b}^{\dagger} \hat{b}\right\rangle>0
$$

However, to detect entanglement this expression should attain negative values. Since the expression is positive, it does not detect entanglement in the states.

5. It is known that $\mathrm{SU}(1,1)$ 31, 32] uncertainty relation can detect entanglement in a class of two-mode non-Gaussian states involving the ground and the first excited states of harmonic oscillator. For the states in any of the invariant subspaces, the criterion states that if

$$
\begin{aligned}
f_{11}= & {\left[1+2 N_{a b}+N_{a}+N_{b}-2 N_{a} N_{b}\right]^{2}-4\left[\Re\left\langle\hat{a}^{2} \hat{b}^{\dagger 2}\right\rangle^{2}-\Re\left\langle\hat{a}^{\dagger} \hat{b}\right\rangle^{2}\right]^{2} } \\
& -\left.\left|N_{a}+N_{b}\right\rangle\right|^{2}<0,
\end{aligned}
$$

the corresponding state is entangled. Here $N_{a b}$ is the expectation value $\left\langle\hat{a}^{\dagger} \hat{a} \hat{b}^{\dagger} \hat{b}\right\rangle$ and $\Re$ stands for real part of the expression that follows.

Simiarly, Simon's criterion [28] identifies entangled states in the invariant subspaces if

$$
\begin{aligned}
f_{S}= & \frac{\Re\left\langle\hat{a} \hat{b}^{\dagger}\right\rangle^{2}}{2}+\left|\left\langle\hat{a} \hat{b}^{\dagger}\right\rangle\right|^{4}-\left|\left\langle\hat{a} \hat{b}^{\dagger}\right\rangle\right|^{2}\left[N_{a}+N_{b}+2 N_{a} N_{b}\right] \\
& +\frac{1}{16}\left(1+2 N_{a}\right)^{2}\left(1+2 N_{b}\right)^{2}<0 .
\end{aligned}
$$

The criterion due to Hillery and Zubairy [33] states that if

$$
f_{H Z}=N_{a} N_{b}-\left|\left\langle\hat{a}^{\dagger} \hat{b}\right\rangle\right|^{2}<0
$$


then the state is entangled. For the states generated when the system evolves from the initial condition $|2,2\rangle$, these three criteria are evaluated numerically. The results are shown in Fig 4, It is seen that the entanglement is not identified by the criteria $f_{H Z}$ and $f_{S}$. The $\mathrm{SU}(1,1)$ criterion detects entanglement in at least some of the states occurring during evolution. This is the reason for choosing the $|2,2\rangle$ as the initial state. If any other product state in $S_{4}$ is chosen as the initial condition, all the three criteria fail.

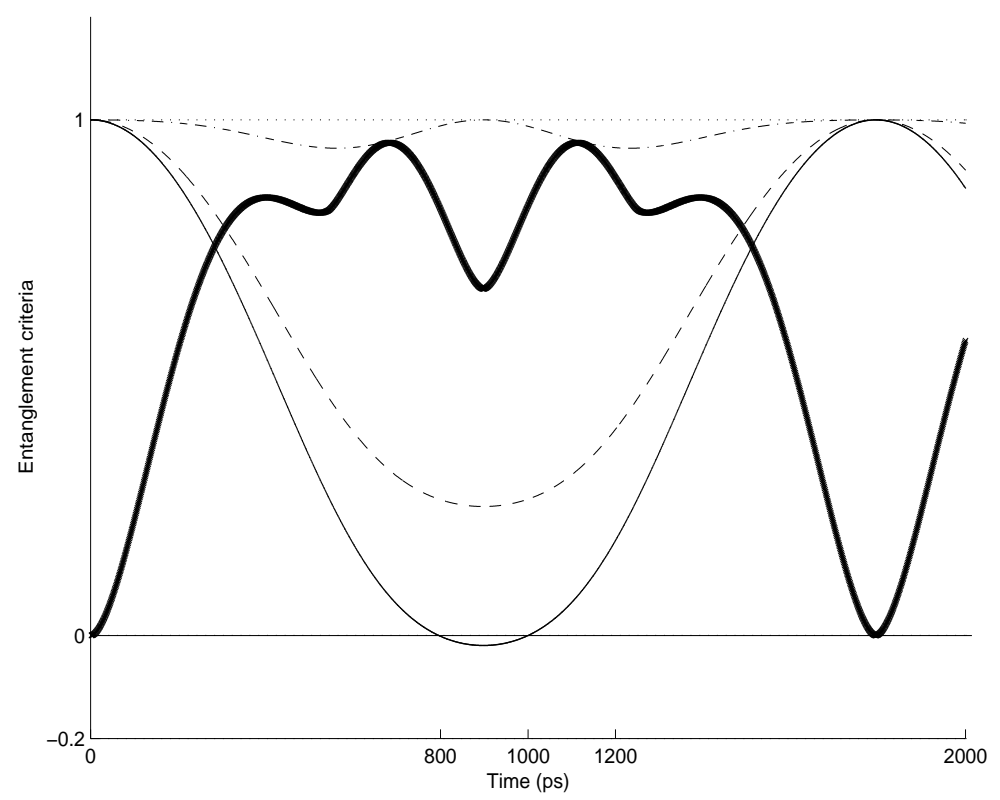

Figure 4: Temporal evolution of criteria for entanglement as the system evolves from the initial state $|2,2\rangle$. The criteria shown are $f_{H S}$ (dashed), $f_{S}$ (dash-dot) and $f_{11}$ (thin continuous). For comparison, the von Neumann entropy is shown (thick continuous). The curves correspond to ratio of the criterion to its absolute maximum value during the evolution. This quantity has been chosen so that the various criteria are limited to a maximum value of one. This ratio is sufficient as it is the occurrence of negative value that is of importance and not actual values of the criteria. The value of $f_{11}$ falls below zero (the horizontal line) between 800 ps to 1000 ps indicating entanglement during those instants. At other instants, the criterion does not detect entanglement. Values of $\gamma, \epsilon$ and $\omega$ are as indicated in Fig. 1.

The failure of the known criteria to detect entanglement provides motivation to look for a new criterion. The states in an invariant subspace are spanned by states of fixed total quantum number $N=n+m$, leading to strong correlation between the number of quanta of the first mode and that of the second mode. It is, therefore, natural to expect that a correlation function of the number operators of the two modes may detect entanglement. Such a criterion for entanglement 
is derived as follows. For all product states the equality $\left\langle\hat{a}^{\dagger} \hat{a} \hat{b}^{\dagger} \hat{b}\right\rangle=\left\langle\hat{a}^{\dagger} \hat{a}\right\rangle\left\langle\hat{b}^{\dagger} \hat{b}\right\rangle$ holds. Consider the most general state $\sum c_{n}|n, N-n\rangle$, where the summing index $n$ runs from zero to $N$. For these states, $\left\langle\hat{a}^{\dagger} \hat{a} b^{\dagger} \hat{b}\right\rangle=N\left\langle\hat{a}^{\dagger} \hat{a}\right\rangle-\left\langle\hat{a}^{\dagger} \hat{a} \hat{a}^{\dagger} \hat{a}\right\rangle$ and $\left\langle\hat{a}^{\dagger} \hat{a}\right\rangle\left\langle\hat{b}^{\dagger} \hat{b}\right\rangle=N\left\langle\hat{a}^{\dagger} \hat{a}\right\rangle-\left\langle\hat{a}^{\dagger} \hat{a}\right\rangle^{2}$. But, $\left\langle\hat{a}^{\dagger} \hat{a} \hat{a}^{\dagger} \hat{a}\right\rangle \geq\left\langle\hat{a}^{\dagger} \hat{a}\right\rangle^{2}$ for any state. Comparing the expressions for $\left\langle\hat{a}^{\dagger} \hat{a} \hat{b}^{\dagger} \hat{b}\right\rangle$ and $\left\langle\hat{a}^{\dagger} \hat{a}\right\rangle\left\langle\hat{b}^{\dagger} \hat{b}\right\rangle$ yields the inequality $\left\langle\hat{a}^{\dagger} \hat{a} \hat{b}^{\dagger} \hat{b}\right\rangle<$ $\left\langle\hat{a}^{\dagger} \hat{a}\right\rangle\left\langle\hat{b}^{\dagger} \hat{b}\right\rangle$. The equality sign holds if $\left\langle\hat{a}^{\dagger} \hat{a} \hat{a}^{\dagger} \hat{a}\right\rangle=\left\langle\hat{a}^{\dagger} \hat{a}\right\rangle^{2}$, which is true if all but one of the $c_{n}$ s is zero. In that case the state is a product state and there is no entanglement. To bring out these features, the temporal variation of the number correlation function $D=\left\langle\hat{a}^{\dagger} \hat{a} \hat{b}^{\dagger} \hat{b}\right\rangle-\left\langle\hat{a}^{\dagger} \hat{a}\right\rangle\left\langle\hat{b}^{\dagger} \hat{b}\right\rangle$ is shown in Fig.5. Negative value of $D$ implies that the state is entangled. For comparison, the von Neumann entropy is shown. It is seen that whenever the entropy is positive, which indicates entanglement, the quantity $D$ is negative. Thus, $D$ qualifies as a suitable criterion to detect entanglement in the class of non-Gaussian, pure states considered.

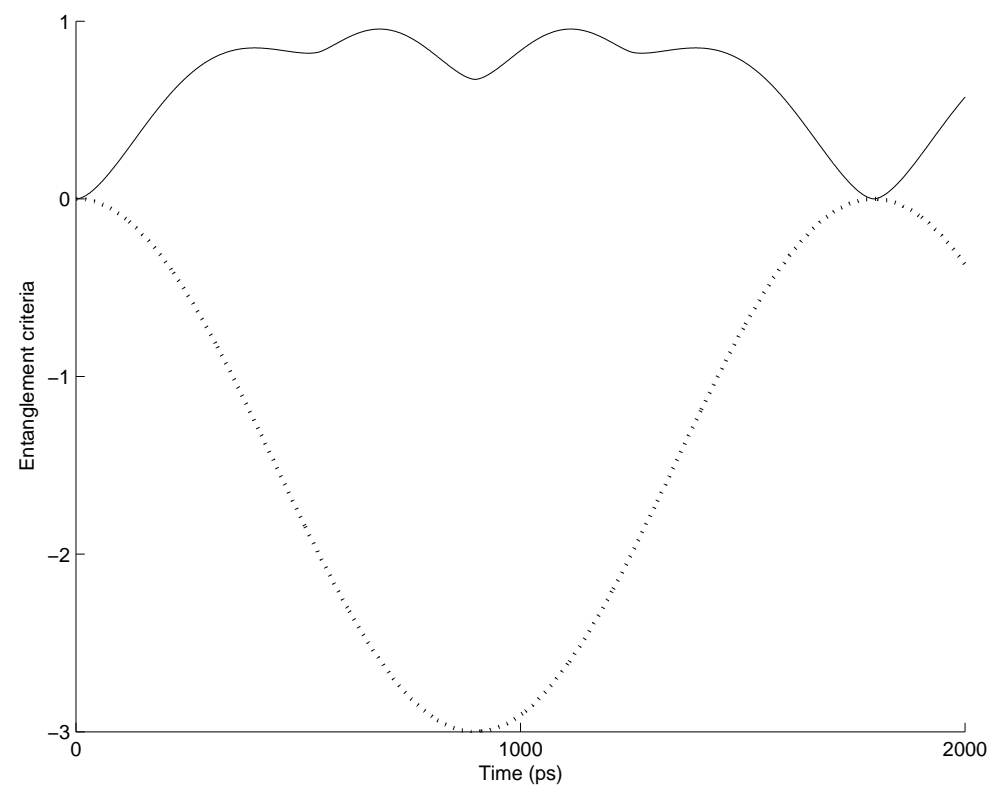

Figure 5: Temporal evolution of criteria for entanglement as the system evolves from the initial state $|2,2\rangle$. The quantities plotted are von Neumann entropy (conitnuous) and $D$ (dashed). Values of $\gamma, \epsilon$ and $\omega$ are as indicated in Fig. 1.

\section{Quadrature variances}

The coupled C-H oscillators modelled by $\hat{H}$ are oscillating masses. The quadratures of these oscillators are naturally identified with their positions and momenta. For the individual oscillators 
described by $\hat{H}$, the position and momentum quadratures are given by

$$
\begin{aligned}
& \hat{Q}_{a}=\frac{\hat{a}+\hat{a}^{\dagger}}{\sqrt{2}}, \\
& \hat{P}_{a}=\frac{\hat{a}-\hat{a}^{\dagger}}{i \sqrt{2}},
\end{aligned}
$$

respectively and they satisfy $\left[\hat{Q}_{a}, \hat{P}_{a}\right]=i$, where $\hbar$ is set equal to unity. The corresponding variances satisfy $2 \Delta Q_{a} \Delta P_{a} \geq 1$. If the variance of one of the quadratures is less than $1 / 2$, the corresponding quadrature is said to exhibit squeezing. Evaluation of the variances in the present case is simplified since the expectation values of operators involving creation and annihilation operators with unequal exponents, vanish in the states within an invariant subspace. For instance, the expectation value of $\hat{a} \hat{a}^{\dagger 2}$ is zero as creation and annihilation operators occur with different exponents; similarly the expectation values of $\hat{a} \hat{b}, \hat{a}^{\dagger} \hat{b}^{\dagger}$, etc all vanish for the states in $S_{N}$. Hence, for the states belonging to $S_{N}$,

$$
\Delta Q_{a}=\sqrt{\frac{1}{2}[1+N]},
$$

and

$$
\Delta P_{a}=\sqrt{\frac{1}{2}[1+N]} .
$$

The suffix $a$ labels the mode. Similar expressions can be written for the other mode and the results on variances are identical to those of the $a$-mode. If $N>0$ the quadratures of the individual oscillators do not exhibit any squeezing for the states in the invariant subspaces. Further, the numerical values of the variances in the two quadratures are equal and increases with the total quantum number $N$. The state $|0,0\rangle$ does not exhibit squeezing and it corresponds to minimum uncertainty state. The notion of quadratures associated with the individual modes has been generalized to multi-mode cases. For the bipartite system, the two-mode quadratures are defined as 34$]$

$$
\begin{aligned}
& \hat{d}_{1}=\frac{\hat{a}+\hat{b}+\hat{a}^{\dagger}+\hat{b}^{\dagger}}{2^{\frac{3}{2}}}, \\
& \hat{d}_{2}=\frac{\hat{a}+\hat{b}-\hat{a}^{\dagger}-\hat{b}^{\dagger}}{i 2^{\frac{3}{2}}} .
\end{aligned}
$$

Based on these expressions for the two-mode quadratures, the respective variances of the quadratures in any state belonging to $S_{N}$ are given by

$$
\begin{aligned}
& \left(\Delta d_{1}\right)^{2}=\frac{1}{4}\left[1+N+\left\langle\hat{a} \hat{b}^{\dagger}\right\rangle+\left\langle\hat{a}^{\dagger} \hat{b}\right\rangle\right] \\
& \left(\Delta d_{2}\right)^{2}=\frac{1}{4}\left[1+N+\left\langle\hat{a} \hat{b}^{\dagger}\right\rangle+\left\langle\hat{a}^{\dagger} \hat{b}\right\rangle\right] .
\end{aligned}
$$


The states belong to the invariant subspace $S_{N}$ possess equal two-mode quadrature variances. The two-mode quadratures satisfy the commutation relation $\left[d_{1}, d_{2}\right]=i / 2$ and the corresponding uncertainty relation is $\Delta d_{1} \Delta d_{2} \geq 1 / 4$. If any of the quadrature has uncertainty lower than $\frac{1}{2}$, the quadrature is said to be squeezed. The fact that the two-mode variances are equal implies that one of the variances cannot be reduced to values less than $1 / 2$, the condition for squeezing, without violating the uncertainty relation. In short, squeezed fluctuations in the single-mode or two-mode quadratures are not possible for the states in any of the invariant subspaces. Though there is no squeezing, the states in the invariant subspaces are very much nonclassical in the sense that their respective $P$-distributions are highly singular.

\section{Generation of maximally entangled states}

Bell states enjoy a special status in quantum information theory as they are maximally entangled bipartite states[1]. The canonical Bell states are the linear superpositions $|0,0\rangle \pm \mid 1,1$ and $|0,1\rangle \pm$ $|1,0\rangle$. Bell-like states are similar combinations allowing for a relative phase between the superposed states. Though the $\mathrm{C}-\mathrm{H}$ oscillators are not two level systems, the presence of invariant subspaces allows for the creation of Bell-like states for the two coupled oscillators. This special feature is available in the invariant subspace $S_{1}$ spanned by $|0,1\rangle$ and $|1,0\rangle$. The Hamiltonian $\hat{H}_{2}$ required to describe the dynamics of the states in the subspace $S_{2}$ is

$$
\hat{H}_{2}=\left[\begin{array}{cc}
\omega-\gamma & -\epsilon \\
-\epsilon & \omega-\gamma
\end{array}\right] .
$$

The eigenvalues of the $\hat{H}_{2}$ are $\omega-\gamma \pm \epsilon$ and the corresponding eigenstates are the Bell states $| \pm\rangle=[|0,1\rangle \pm|1,0\rangle] / \sqrt{2}$.

If the initial state is $|0,1\rangle=[|+\rangle+|-\rangle] / \sqrt{2}$ then

$$
\exp \left(-i t \hat{H}_{2}\right)|0,1\rangle \propto(1+\exp (i \epsilon t))|0,1\rangle+(1-\exp (i \epsilon t))|1,0\rangle
$$

apart from an overall multiplicative normalization factor. If the time $t$ is chosen to be $\epsilon t=\pi / 2$, then the time-evolved state is the Bell-like state $|0,1\rangle-i|1,0\rangle$, apart from an overall phase factor. If $\epsilon t=3 \pi / 2$, the initial state evolves to become another Bell-like state $|0,1\rangle+i|1,0\rangle$, but for an overall phase factor. The overlap between the time-evolved state of the system and the Bell-like states are given in Fig. 6. At specific instants, the initial state evolves to have unit overlap with $|0,1\rangle+i|1,0\rangle$; there is an overall phase factor to the actual state achieved and the overlap is insensitive to such factors. Since the subspace $S_{1}$ is invariant under the unitary evolution, the other two Bell-like states, $|0,0\rangle \pm|1,1\rangle$ are not attainable with any initial condition contained in $S_{1}$. These states belong to the direct sum of $S_{0}$ and $S_{2}$. Without external control fields it is not feasible to generate all the Bell-like states using the inherent coupling in the system. 


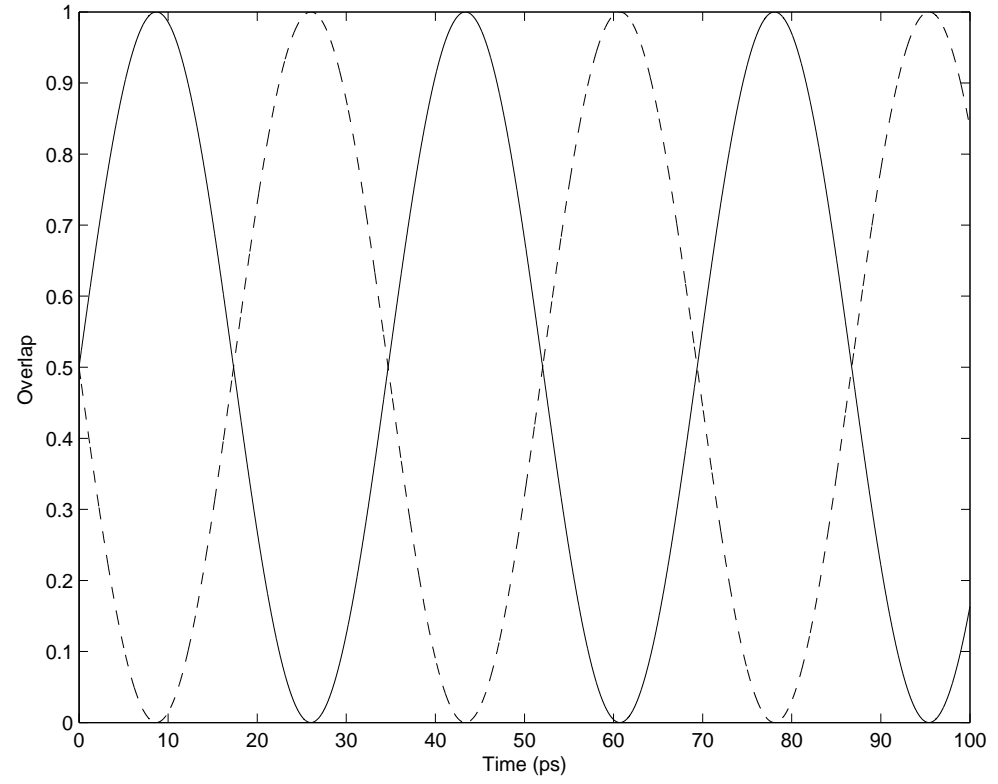

Figure 6: Overlap between the state $\exp \left(-i t \hat{H}_{2}\right)|0,1\rangle$ and the Bell-like states $|0,1\rangle+i|1,0\rangle$ (continuous) and $|0,1\rangle-i|1,0\rangle$ (dashed). The instants when the overlap becomes unity, the initial state has evolved into the corresponding Bell-like state. Values of $\gamma, \epsilon$ and $\omega$ are the same as for Fig. 1 


\section{Summary}

The Hamiltonian for the coupled, nonlinear C-H oscillators of dihalomethanes is similar to that of the nonlinear couplers with negative $\chi^{2}$. Thus the $\mathrm{C}-\mathrm{H}$ oscillators are the microscopic nonlinear couplers for bosonic fields of vibrational-cum-bending motion. Like the Kerr nonlinear couplers, the dihalomethane can serve as a system wherein entanglement is easily generated with the advantage that there is no need to have external fields for the purpose of entangling the modes. The coupling due to the bending motion of the molecule creates entanglement between the modes and allows for exchange of energy. The structure of the Hamiltonian with commuting terms allows to split the Hilbert space into irreducible, invariant subspaces. Each of the invariant subspace is the span of the product states of the form $|n, m\rangle$ with $n+m$ fixed for the individual subspaces. Apart from the canonical basis states, the remaining states in the invariant subspaces are entangled. The states belonging to the invariant subspaces are non-Gaussian states. The know detection criteria for entangled states do not identify entanglement in the states belonging to the invariant subspaces. However, for these non-Gaussian pure states the entanglement criterion is that $\left\langle\hat{a}^{\dagger} \hat{a} \hat{b}^{\dagger} \hat{b}\right\rangle\left\langle\left\langle\hat{a}^{\dagger} \hat{a}\right\rangle\left\langle\hat{b}^{\dagger} \hat{b}\right\rangle\right.$.

The local modes of sufficient energy are approximate eigenstates of the complete Hamiltonian. The entanglement in the evolved state is much larger if the initial state is different from a local mode. Though there is a change of quadrature variance during evolution, the states in the invariant subspaces do not exhibit single-mode or two-mode quadrature squeezing. The variances are always above the minimum allowed limit. The two eigenstates of the total Hamiltonian, belonging to the subspace $S_{1}$ correspond to two of the Bell states. Though states of high entanglement cannot be generated from the local modes of high energy, the Hamiltonian generates maximally entangled states during the evolution of the local modes $|0,1\rangle$ or $|1,0\rangle$.

\section{References}

[1] M. A. Nielsen and I. L. Chuang Quantum Computation and Quantum Information (Cambridge: Cambridge University Press) (2000)

[2] G. Chen et al Quantum Devices Principles, Design, and Analysis (USA: Chapman \& Hall) (2007)

[3] C. M. Tesch, L. Kurtz and R. de Vivie-Riedle Chemical Physics Letters 343(2001) 633

[4] D. Babikov Journal of Chemical Physics 121 (2004) 7577

[5] A. S. Scott Nonlinear Science: Emergence and Dynamics of Coherent Structures, (London: Oxford University Press)(2003)

[6] A. Chefles and S. M. Barnett Journal of Modern Optics 43 (1996) 709 
[7] L. J. Bernstein Physica D 68 (1993) 174

[8] S L Braunstein and P van Loock Reviews of Modern Physics 77 (2005) 513

[9] A. Furusawa et al Science 282 (1998) 706

[10] E. Ciancio and P. Zanardi Physics Letters A 360 (2006) 49

[11] J. Perina Quantum Statistics of linear and nonlinear optical phenomena (The Netherlands: Kluwer Academic Publishers)(1991)

[12] G. S. Agarwal and R. R. Puri Physical Review A 39 (1989) 2969

[13] S. M. Jensen IEEE J. Quantum Electron. QE-18 (1982) 1580

[14] A. Miranowicz and W. Leonski Journal of Physics B: Atomic, Molecular and Optical Physics 39 (2006) 1683

[15] W. Leonski and A. Miranowicz Journal of Optics B 6 (2004) S37.

[16] L. Sanz, R. M. Angelo and K. Furuya Journal of Physics A: Mathematical and General 36 (2003) 9737

[17] G. Ariunbold and J. Perina Journal of Modern Optics 48 (2001) 1005

[18] M. K. Olsen Physical Review A 73 (2006) 053806

[19] C. Sudheesh, S. Lakshmibala and V. Balakrishnan Journal of Physics B: Atomic,Molecular and Optical Physics 39 (2006) 3345

[20] A. Kowalewska-Kudlaszyk and W. Leonski Physical Review A 73 (2006) 042318

[21] C. H. Bennett, H. J. Bernstein, S. Popescu and B. Schumacher Physical Review A 53 (1996) 2046

[22] M. S. Kim, W. Son, V. Buzek and P. L. Knight Physical Review A 65 (2002) 032323

[23] Wang Xiang-bin Physical Review A 66 (2002) 024303

[24] L. Mandel and E. Wolf Optical Coherence and Quantum Optics (Cambridge: Cambridge University Press)(1998)

[25] N. Korolkova and J. Perina Optics Communications 136 (1996) 135

[26] G. C. Gerry and P. L. Knight Introductory Quantum Optics (Cambridge: Cambridge University Press) (2004) 
[27] L.-M. Duan et al Physical Review Letters 84 (2000) 2722

[28] R. Simon Physical Review Letters 84 (2000) 2726

[29] S. Mancini et al Physical Review Letters 88 (2002) 120401

[30] E. Shchukin and W. Vogel Physical Review Letters 95 (2005) 230502

[31] G. S. Agarwal and A. Biswas New Journal of Physics 7 (2005) 211

[32] H. Nha and J. Kim Physical Review A 74 (2006) 012317

[33] M. Hillery and M. S. Zubairy Physical Review Letters 96 (2006) 050503

[34] C. M. Caves Physical Review D 26 (1985) 1817 